\title{
Articles
}

\section{Maternal dietary vitamin restriction increases body fat content but not insulin resistance in WNIN rat offspring up to 6 months of age}

\author{
L. Venu ${ }^{1}$ N. Harishankar ${ }^{2}$ T. Prasanna Krishna ${ }^{3}$ M. Raghunath ${ }^{1}$ \\ ${ }^{1}$ Endocrinology and Metabolism Division, National Institute of Nutrition (ICMR), Hyderabad, India \\ 2 National Centre for Laboratory Animal Sciences, National Institute of Nutrition (ICMR), Hyderabad, India \\ ${ }^{3}$ Division of Statistics, National Institute of Nutrition (ICMR), Hyderabad, India
}

\begin{abstract}
Aims/hypothesis. Epidemiological evidence suggests that some adult diseases like insulin resistance syndrome and diseases associated with it originate in fetal life. The role of maternal macronutrient malnutrition but not of micronutrients in the fetal origin of adult disease is well studied. We hypothesise that chronic maternal vitamin restriction predisposes the offspring to insulin resistance syndrome.

Methods. Female weanling Wistar/NIN rats received a control diet $(n=6)$ or a $50 \%$ vitamin-restricted diet $(n=14)$ for 12 weeks and mated with control males. Four dams on the restricted diet were shifted to the control diet from parturition. Pups born to the remaining 10 dams on the restricted diet were weaned on to control diet or continued on the restricted diet. All groups had 8 male pups from weaning onwards.

Results. Birthweights of pups were comparable among different groups. Weaning body weights were low in the restricted diet group, but on rehabilitation they
\end{abstract}

caught up with control animals by post-natal day 100 . None of the pups had impaired oral glucose tolerance and their insulin resistance status was comparable on days 40, 70, 100 and 180. Compared with offspring on the control diet, offspring on the restricted diet had a significantly higher percentage of body fat and higher plasma triglycerides, as well as lower lean body mass and fat-free mass. They also had increased oxidative stress. Rehabilitation from parturition or weaning prevented the changes in body fat percent, lean body mass, fat-free mass and oxidative stress.

Conclusions/interpretation. Since changes in adiposity and fat metabolism are considered forerunners of insulin resistance syndrome, our observations suggest that maternal dietary vitamin restriction predisposes the offspring to insulin resistance syndrome in later life.

Keywords Body fat $\cdot$ Glucose tolerance $\cdot$ Insulin resistance $\cdot$ Maternal undernutrition - Oxidative stress $\cdot$ Rehabilitation $\cdot$ Triglycerides $\cdot$ Vitamins
Received: 4 February 2004 / Accepted: 28 June 2004

Published online: 10 September 2004

(C) Springer-Verlag 2004

M. Raghunath (『)

Endocrinology and Metabolism Division,

National Institute of Nutrition (ICMR),

Jamai Osmania P O, Hyderabad-500 007, India

E-mail: manchalar@yahoo.com

Tel.: +91-40-27008921 ext 235, Fax: +91-40-27019074

Abbreviations: GPx, glutathione peroxidase $\cdot$ GSH, reduced glutathione - HOMA-IR, homeostasis model assessment of insulin resistance $\cdot$ MDA, malondialdehyde $\cdot$ SOD, superoxide dismutase - TOBEC, total body electrical conductivity · VSP, vitamin-restricted mothers and their pups receiving vitamin supplementation from parturition and weaning respectively . VSW, vitamin-restricted offspring weaned on to control diet . WNIN, Wistar/NIN

\section{Introduction}

Intrauterine and early post-natal malnutrition has profound consequences on fetal and post-natal development in humans and animals $[1,2]$. Low birthweight is associated [3] with increased risk of the metabolic syndrome in adulthood. Metabolic syndrome, a cluster of cardiovascular risk factors such as diabetes, hypertension, dyslipidaemia and obesity, is now a recognised major health problem in developing countries like India [4]. Insulin resistance is the common underlying feature, but its causes are not completely understood. These observations have led to the hypothesis that intrauterine growth retardation affecting fetal development predisposes the offspring to insulin resistance and diabetes in later life $[5,6]$. 
Maternal nutrition directly affects fetal growth and development by modulating the availability of nutrients for transfer to the fetus. It may also permanently alter glucose and insulin metabolism [7]. Several metabolic abnormalities that lead to insulin resistance have been demonstrated in the offspring of rat dams fed protein- or energy-restricted diets [7]. Most studies reported so far have considered only maternal deficiency of macronutrients, but not of micronutrients.

Micronutrients such as vitamins and minerals play essential roles in cellular metabolism, maintenance and growth throughout life. Several studies indicate that vitamins $\mathrm{A}, \mathrm{D}, \mathrm{E}, \mathrm{C}$ and folate affect insulin sensitivity [8]. Moreover, deficiencies of these vitamins can have profound and often persistent effects on many fetal tissues and organs, even in the absence of any clinical signs of deficiency in the mother [9]. Furthermore, the consequences of vitamin imbalance during fetal development may not be apparent at the time of the nutritional insult, but may only become manifest during later development [9]. Multiple vitamin deficiencies, particularly during pregnancy and/or lactation, are common in the developing world and maternal vitamin deficiencies are associated with low birthweights and increased rates of perinatal mortality and morbidity [10]. Interestingly, the prevalence of low birthweight in developing countries varies from $13 \%$ to $30 \%$ [11]. Despite this and the known effects of vitamins per se on insulin action, the role of maternal vitamin deficiencies in the development of insulin resistance in the offspring has not been assessed so far.

It could, however, be of great interest in elucidating why the incidence of metabolic syndrome and Type 2 diabetes is increasing in the Indian population. In view of the literature cited above, we hypothesised that maternal dietary vitamin restriction predisposes the offspring to insulin resistance in later life. Our study was carried out in Wistar/NIN (WNIN) rats to validate or negate this hypothesis.

\section{Materials and methods}

Feeding, maintenance and breeding of animals. All animal experiment procedures were carried out in accordance with the 'principles of laboratory animal care' (NIH publication no. 85-23, revised 1985) and with the approval of the ethical committee on animal experiments at National Institute of Nutrition, Hyderabad, India.

Female, weanling Wistar/NIN (WNIN) rats $(n=20)$ were obtained from National Centre for Laboratory Animal Sciences, National Institute of Nutrition, Hyderabad, India. They were divided into 2 groups of 6 and 14, housed individually in polypropylene cages with wire mesh bottom and maintained at $22 \pm 2{ }^{\circ} \mathrm{C}$, under standard lighting conditions (12-h light/dark cycle). For 12 weeks the group of 14 rats was fed a basal diet (AIN-93G) [12], with $50 \%$ restriction of vitamin mixture (composition of diet and vitamin mixture, see Table 1). The other group of 6 rats served as the pair-fed control for the vitamin-restricted group. All the animals were provided deionised
Table 1. Composition of animal diets (ingredients in $\mathrm{g} / \mathrm{kg}$ )

\begin{tabular}{lcc}
\hline Ingredient & $\begin{array}{l}\text { Control } \\
\text { diet }\end{array}$ & $\begin{array}{l}50 \% \text { vitamin- } \\
\text { restricted diet }\end{array}$ \\
\hline Corn starch & 487 & 492 \\
Casein ( 70\% protein) & 243 & 243 \\
Sucrose & 100 & 100 \\
Soya bean oil & 70 & 70 \\
Cellulose & 50 & 50 \\
Mineral mix (AIN-93G-MX) & 35 & 35 \\
Vitamin mix (AIN-93-VX) & 10 & 5 \\
L-cystine & 3 & 3 \\
Choline chloride & 2.5 & 2.5 \\
\hline
\end{tabular}

a Contains following vitamins (g/kg mix): nicotinic acid, 3.0; calcium pantothenate, 1.6; pyridoxine- $\mathrm{HCl}, 0.7$; thiamin- $\mathrm{HCl}$, 0.6; riboflavin, 0.6; folic acid, 0.2; biotin, 0.02; vitamin B12, 2.5; vitamin E (500 IU/g), 15.0; vitamin A (500,000 IU/g), 0.8; vitamin D3 (400,000 IU/g), 0.25; vitamin K1, 0.075

water. At the end of 12 weeks of feeding, blood was collected from supra orbital sinus to determine the vitamin status of the rats, in addition to the following biochemical parameters: haemoglobin, glucose, insulin, cholesterol and triglycerides.

After assessment of their vitamin status, the rats were mated with control males ( 2 females to 1 male) and maintained on their respective diets throughout gestation. At parturition, 4 of the vitamin-restricted dams were shifted to control diet (VSP) and the remaining 10 vitamin-restricted dams continued on the restricted diet throughout lactation. During lactation, a uniform litter size was maintained in all groups from post-natal day 3, by adjusting the number of offspring per litter to 8 (equal number of male and female pups with most mothers). From weaning (post-natal day 21 ), only 8 male pups, derived from 4-5 mothers of the corresponding group, were maintained in each of the groups till post-natal day 180 . While control-diet pups remained on the control diet, VSP pups and 8 vitamin-restricted pups were weaned on to control diet (VSW), with another 8 vitaminrestricted pups continuing on the restricted diet. To avoid the possible effects of estrus cycle on glucose and fat metabolism and insulin resistance, only male offspring have been included in this study. The feeding protocol used in this experiment is depicted schematically in Figure 1. Daily diet intake and weekly body weights were recorded in mothers and offspring.

Measurements in plasma. Blood was collected from offspring at 40, 70, 100 and 180 days of age following an overnight fast; plasma was separated and stored at $-20{ }^{\circ} \mathrm{C}$ till analysis. Vitamins $\mathrm{A}$ and $\mathrm{E}$ were measured in maternal plasma by HPLC [13] and folic acid by radioimmunoassay using a commercially available kit (Diagnostic Products, Los Angeles, Calif., USA), according to the manufacturer's instructions.

Plasma glucose and HDL cholesterol were measured enzymatically using kits (Dr. Reddy Labs, Hyderabad, India) according to the manufacturer's instructions. Total cholesterol and triglycerides were measured in fasting plasma using enzymatic kits (Biosystems, Barcelona, Spain). Plasma insulin was measured using the radioimmunoassay kit from BRIT, Mumbai, India.

Oral glucose tolerance test. An OGTT was performed in 6 to 8 pups of all 4 groups on post-natal days 40, 70, 100 and 180 . After an overnight fast, glucose (300 g/l) was administered orogastrically at a dose of $2.5 \mathrm{~g} / \mathrm{kg}$ body weight and blood samples 


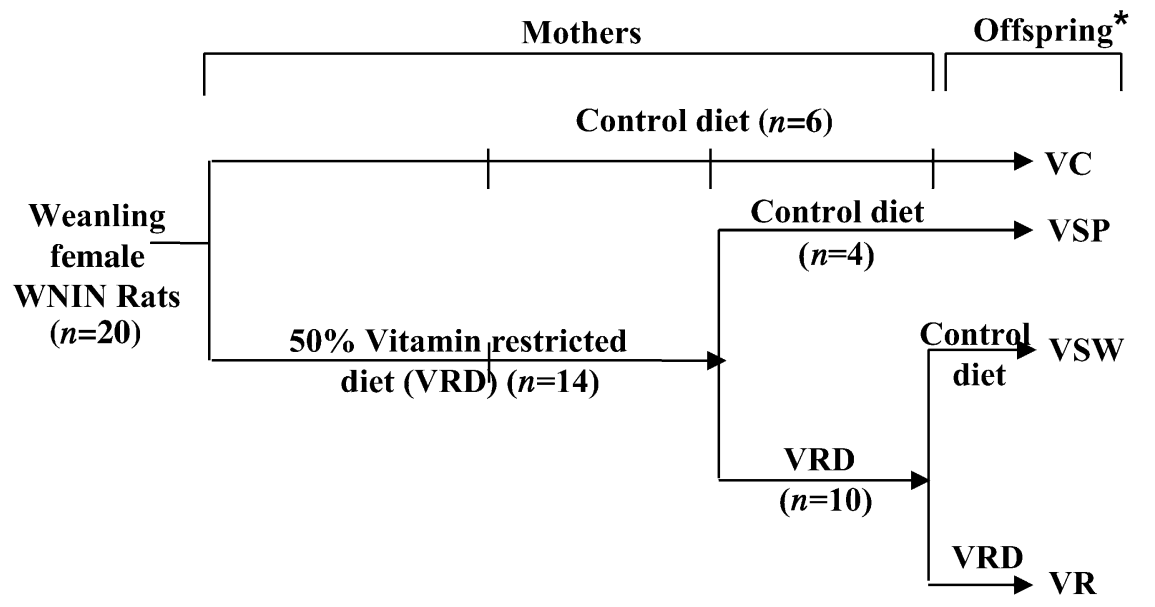

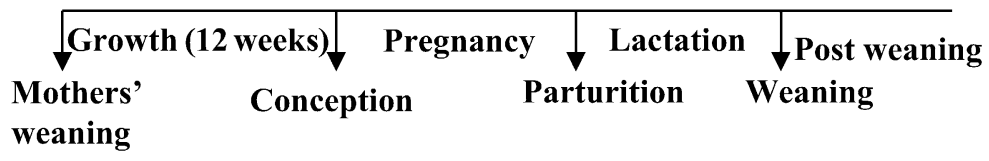

Fig. 1. Schematic representation of the feeding protocol of different groups of mothers and the offspring. VC, control diet throughout; VR, vitamin restriction throughout; VSP, vitamin supplementation to vitamin-restricted mothers from parturition and to pups of such mothers from weaning; VSW, vitamin-restricted offspring weaned on to control diet. $* n=8$ male offspring in each group from weaning

were obtained from supra orbital sinus at 0,60 and 120 min to determine plasma glucose and insulin concentrations. Glucose and insulin responses during the OGTT were calculated by computation of the total area under the glucose and insulin curves respectively, using the trapezoidal method [14].

Physiological indices of insulin resistance and glucose tolerance. Indices were based on fasting glucose, insulin concentrations calculated according to the homeostasis model assessment for insulin resistance (HOMA-IR) and on the animals' response to an oral glucose challenge (AUC glucose / AUC insulin). We computed the indices as follows:

1. HOMA-IR. Insulin resistance was assessed from fasting glucose and fasting insulin concentrations using the following formula:

$$
\begin{aligned}
H O M A-I R= & (\text { fasting insulin }[\mu U / \mathrm{ml}) \\
& \times \text { fasting glucose }[\mathrm{mmol} / \mathrm{l}]) / 22.5
\end{aligned}
$$

2. Glucose AUC : insulin AUC. The AUCs during the OGT were calculated by the trapezoidal method [14] and the glucose AUC : insulin AUC was computed.

Body composition of the offspring. Total body composition of the offspring was determined on post-natal days 100 and 180 using the Total Body Electrical Conductivity (TOBEC) small animal body composition analysis system (EM-SCAN, Model SA-3000 Multi detector, Springfield, Ill., USA). This instrument determines the total body electrical conductivity of small animals in a non-destructive fashion. The difference between the impedance measured when the animal is inside the electromagnetic field and when the chamber is empty, is an index of the total electrical conductivity of the body, which in turn is proportional to the animal's lean body mass.
Prior to TOBEC measurement, the rats were anaesthetised lightly with ether and placed in the carrier in such a way that the animal's body was stretched to its maximum comfortable length. Then the carrier was placed in the TOBEC chamber and 10 to 12 recordings/scans were taken for each rat. The highest and lowest readings were excluded and the remaining 10 were averaged. The intra-assay coefficient of variation was less than $3.0 \%$. Prior to the measurements, the instrument was calibrated with a standard coil (ID 3076; supplied with the instrument) and the empty chamber read 0-2 units.

The following body composition parameters were obtained mathematically according to the methods of Morbach and coworkers [15], where $E$ stands for total electrical conductivity:

1. Lean body mass $=0.5 E+(0.3 \times$ total body weight $)$

2. Total body fat $=$ total body weight-lean body mass Total body fat percent

$=($ total body fat/total body weight $) \times 100$

3. Fat-free mass $=16.28+0.4 E$

Oxidative stress and antioxidant status. On post-natal day 180 the animals were killed by decapitation. The livers were dissected out immediately, washed thoroughly with ice-cold $0.9 \%$ $\mathrm{NaCl}$, frozen in liquid nitrogen and stored at $-80{ }^{\circ} \mathrm{C}$ until the analysis.

About $1 \mathrm{~g}$ liver (from the biggest lobe) was weighed out, minced and homogenised $(10 \% \mathrm{w} / \mathrm{v})$ in $50 \mathrm{mmol} / \mathrm{l}$ phosphate buffer $(\mathrm{pH}=7.0)$. The homogenate was centrifuged at $1000 \mathrm{~g}$ for $20 \mathrm{~min}$ at $4{ }^{\circ} \mathrm{C}$. A part of the supernatant was used to estimate lipid peroxidation and protein carbonyls. The remaining supernatant was further centrifuged at $12,000 \mathrm{~g}$ for $20 \mathrm{~min}$ at $4{ }^{\circ} \mathrm{C}$ to obtain the postmitochondrial supernatant. This was used to estimate reduced glutathione (GSH) and activities of the antioxidant enzymes: catalase, superoxide dismutase (SOD) and glutathione peroxidase (GPx).

Lipid peroxidation was measured by the thiobarbituric acid colour reaction for malondialdehyde (MDA) [16]. Protein carbonyl content was measured spectrophotometrically using 2,4-dinitro-phenyl-hydrazine [17]. GSH was determined by its reaction with o-phthalaldehyde [18]. Total SOD activity was assayed by monitoring the rate of inhibition of pyrogallol reduction [19]. One unit of SOD represents the amount of enzyme required for $50 \%$ inhibition of pyrogallol reduction per minute. Catalase was assayed by monitoring the disappearance 
of $\mathrm{H}_{2} \mathrm{O}_{2}$ at $240 \mathrm{~nm}$ [20]. One unit of catalase represents the decrease of $1 \mu \mathrm{mol}$ of $\mathrm{H}_{2} \mathrm{O}_{2}$ per minute. A coupled enzyme procedure, with cumene $\mathrm{H}_{2} \mathrm{O}_{2}$ as a substrate, was used to determine total GPx activity [21]. One unit of GPx was defined as the number of $\mu \mathrm{mol}$ of NADPH oxidised per minute. The protein content was determined using the modified Lowry method [22].

Statistical analysis. Data were subjected to statistical analyses using SPSS statistics package (version 10.0). Differences between the control and restricted mothers and their offspring (from birth to weaning) were analysed using the Student's $t$ test. Data collected from the offspring after weaning were analysed using ANOVA, followed by post hoc tests of the least significance difference. Wherever heterogeneity of variance was observed, differences between groups were tested by nonparametric Mann-Whitney $U$ tests. All values are presented as means \pm SEM. The differences were considered significant at a $p$ value of at least $p<0.05$.

\section{Results}

Parameters in mothers

Maternal growth, vitamin status, glucose tolerance and reproductive performance. Body weight gain in

Table 2. Diet intake, physical and biochemical parameters in WNIN female rats fed control and vitamin-restricted diets for 12 weeks from weaning

\begin{tabular}{lcc}
\hline Parameter & Control & Restricted \\
\hline Food intake (g/day) & $10.4 \pm 0.48$ & $10.9 \pm 0.30$ \\
Body weight gain $(\mathrm{g})$ & $162 \pm 2.80$ & $159 \pm 3.56$ \\
Haemoglobin $(\mathrm{mmol} / \mathrm{l})$ & $7.82 \pm 0.11$ & $7.07 \pm 0.11^{* * *}$ \\
Fasting glucose $(\mathrm{mmol} / \mathrm{l})$ & $4.29 \pm 0.23$ & $4.12 \pm 0.12$ \\
Fasting insulin $(\mathrm{pmol} / \mathrm{l})$ & $413 \pm 43.3$ & $408 \pm 21.0$ \\
HOMA-IR & $12.2 \pm 1.34$ & $12.0 \pm 0.66$ \\
Glucose AUC $\left(\mathrm{mmol} \cdot \mathrm{l}^{-1} \cdot \mathrm{h}^{-1}\right)$ & $13.7 \pm 0.51$ & $13.3 \pm 0.58$ \\
Total cholesterol $(\mathrm{mmol} / \mathrm{l})$ & $1.68 \pm 0.23$ & $1.89 \pm 0.09$ \\
Triglycerides $(\mathrm{mmol} / \mathrm{l})$ & $0.44 \pm 0.06$ & $0.71 \pm 0.09^{*}$ \\
Folic acid $(\mathrm{mmol} / \mathrm{l})$ & $449 \pm 17.2$ & $431 \pm 20.6$ \\
Vitamin A $(\mu \mathrm{mol} / \mathrm{l})$ & $1.29 \pm 0.07$ & $1.26 \pm 0.06$ \\
Vitamin E $(\mu \mathrm{mol} / \mathrm{l})$ & $46.3 \pm 1.93$ & $39.7 \pm 1.4 *$ \\
\hline
\end{tabular}

Values represent means \pm SEM of 6 rats per group. $* p<0.05$, $* * * p<0.001$ by Student's $t$ test
WNIN female rats was not affected by feeding vitamin-restricted diets for 3 months from weaning (Table 2) compared to pair-fed control animals, although haemoglobin levels were significantly decreased $(p<0.001)$. The vitamin-restricted diet had no effect on plasma folic acid and vitamin A levels, but vitamin $\mathrm{E}$ (tocopherol) levels were significantly lower in that group than in control animals $(p<0.05)$ (Table 2). Also, there were no differences between the two groups in fasting glucose, insulin and HOMA index or the AUC of glucose during the OGTT. Although plasma total cholesterol was not different between the two groups, plasma triglycerides were significantly higher in vitamin-restricted dams $(p<0.05)$ (Table 2$)$.

Maternal weight gain during pregnancy, litter size and the birthweight of the offspring were comparable between vitamin-restricted and pair-fed control dams. In addition, pup mortality during parturition and lactation did not change significantly on the vitaminrestricted diet.

\section{Parameters in offspring}

Growth characteristics. Despite similar birthweights and the fact that a uniform litter size per mother was maintained from post-natal day 3, weaning weights of vitamin-restricted and VSP pups were significantly lower than those of controls (Table 3 ). While vitaminrestricted offspring continued to weigh less till postnatal day 180, VSP and VSW offspring caught up with controls by post-natal day 180 (Table 3).

Glucose tolerance and insulin resistance. There were no significant differences among different groups in fasting glucose levels till post-natal day 100, but there were some transient changes in vitamin-restricted pups on post-natal day 40. On post-natal day 180, the VSW group had significantly higher fasting glucose levels $(p<0.01)$ than control offspring (Fig. 2a). However, there were no significant differences in fasting insulin levels among the 4 groups at any of the 4 time points tested (Fig. 2b).

Table 3. Body weights of the offspring at weaning and on post-natal days 40, 70, 100 and 180

\begin{tabular}{|c|c|c|c|c|}
\hline Post-natal day & $\mathrm{VC}$ & VR & VSP & VSW \\
\hline 21 (weaning) & $43.5 \pm 0.66^{\mathrm{a}}$ & $35.8 \pm 0.88^{b}$ & $37 \pm 0.69^{b}$ & $35.8 \pm 0.88^{b}$ \\
\hline 40 & $110 \pm 2.54^{\mathrm{a}}$ & $85.3 \pm 3.2^{\mathrm{b}}$ & $91.3 \pm 3.8^{b}$ & $107 \pm 7.2^{\mathrm{a}}$ \\
\hline 100 & $303 \pm 5.1^{\mathrm{a}}$ & $283 \pm 2.83^{b}$ & $315 \pm 5.49^{a}$ & $305 \pm 6.4^{\mathrm{a}}$ \\
\hline 180 & $362 \pm 6.4^{\mathrm{a}}$ & $346 \pm 2.24^{b}$ & $392 \pm 4.84^{c}$ & $351 \pm 7.6^{\mathrm{a}}$ \\
\hline
\end{tabular}

Values given are the mean of observations in 6 rats \pm SEM. $\mathrm{VC}$, control diet throughout; VR, vitamin restriction throughout; VSP, vitamin supplementation to restricted mothers from parturition and to pups of such mothers from weaning; VSW, vitamin-restricted offspring weaned on to control diet. Means with different superscript letters are significantly different by one-way ANOVA ( $F$ values: at 21 days $3.9, p<0.05$; at 40 days $3.56, p<0.05$; at 70 days $3.82, p<0.05$; at 100 days $3.9, p<0.01$; at 180 days $10.59, p<0.05)$ followed by post hoc least significant difference test 

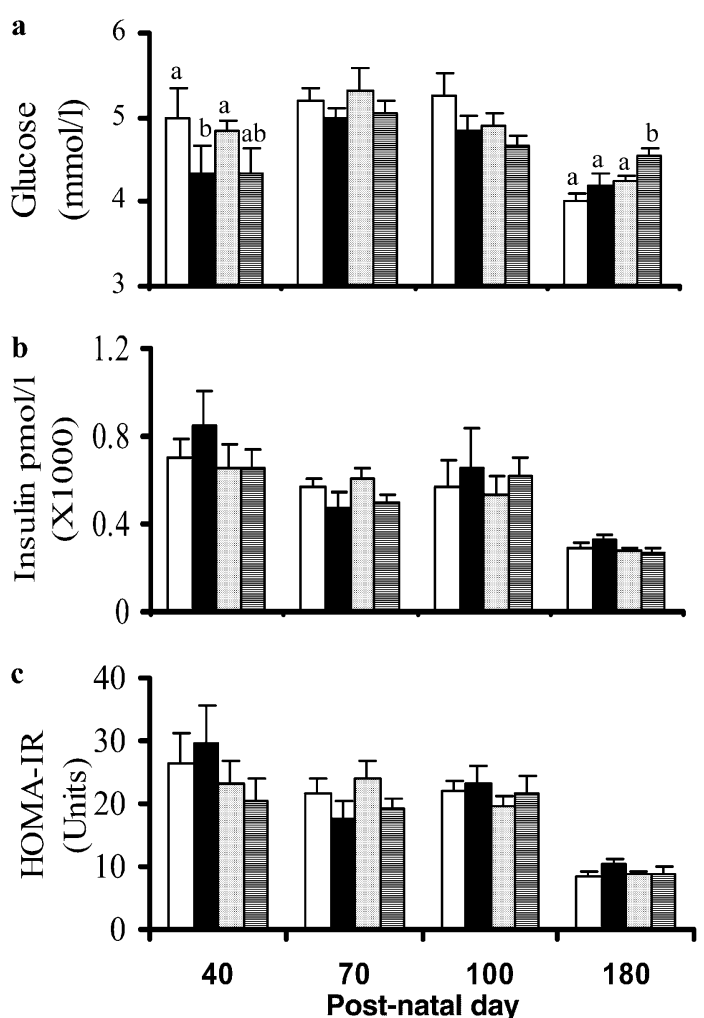

Fig. 2. Fasting plasma glucose (a), insulin levels (b) and HOMA-IR (c) on different post-natal days of the rat offspring of different groups. Open bars: control diet throughout; closed bars: vitamin restriction throughout; lightgrey bars: vitamin supplementation to restricted mothers from parturition and to pups of such mothers from weaning; darkgrey bars: vitaminrestricted offspring weaned on to control diet. Each bar represents a mean of observations in 6 rats. The line above each bar is the SEM. Means with different superscript letters are significantly different by one-way ANOVA ( $F$ value: at 40 days 3.63 , $p<0.05$; at 180 days $3.9, p<0.01)$ followed by post hoc least significant difference test

The AUCs for glucose and insulin during an OGTT in general were not significantly different among the 4 groups till post-natal day 180 (Fig. 3a, b), but there were some transient changes in vitamin-restricted offspring on post-natal days 40 (AUC glucose) and 70 (AUC insulin).

With regard to insulin resistance, there were no significant differences among the groups in the HOMAIR values at any time point tested (Fig. 2c). However, the glucose AUC : insulin AUC ratio was significantly higher in the vitamin-restricted and VSW groups $(p<0.01)$ than in the control and VSP groups at 180 days.

Body composition and plasma lipids. Compared with control offspring, body fat percent was significantly higher $(p<0.01)$ in offspring of vitamin-restricted mothers (Fig. 4a). Other markers of adiposity like lean body mass and fat-free mass (Fig. 4b, c) were signifi-
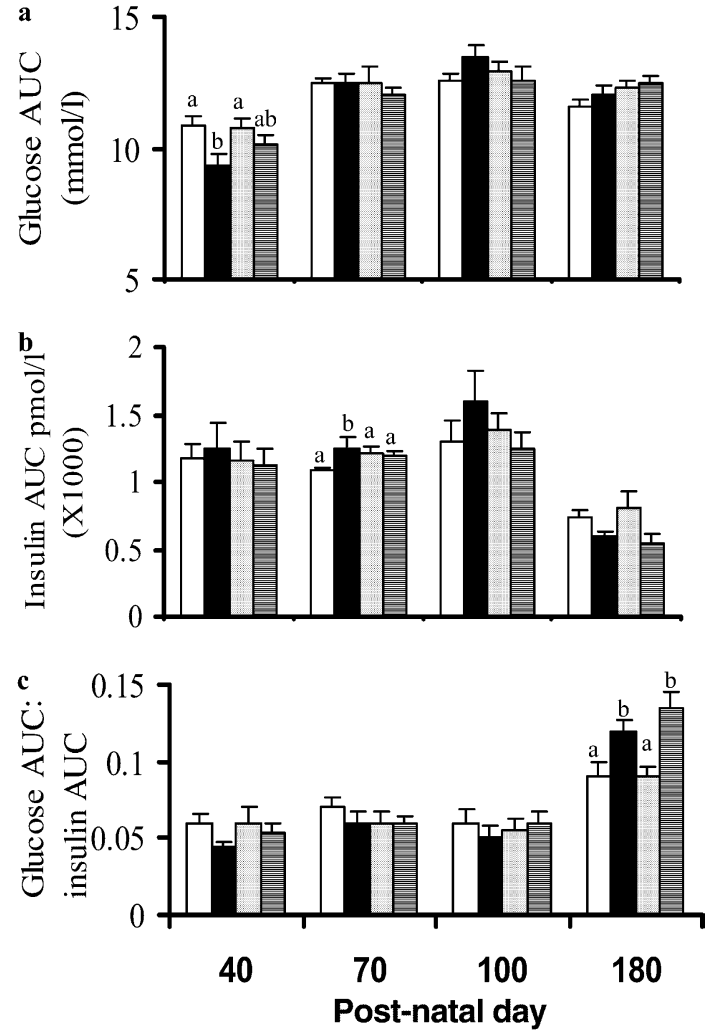

Fig. 3. Area under the curve of plasma glucose (a) and insulin (b), and AUC glucose : AUC insulin ratio (c) during an OGTT on the offspring, conducted on different post-natal days. Open bars: control diet throughout; closed bars: vitamin restriction throughout; lightgrey bars: vitamin supplementation to restricted mothers from parturition and to pups of such mothers from weaning; darkgrey bars: vitamin-restricted offspring weaned on to control diet. Each bar represents a mean of observations in 6 rats. The line above each bar is the SEM. Means with different superscript letters are significantly different by one-way ANOVA ( $F$ values: AUC glucose 4.1, $p<0.05$; AUC insulin $3.52, p<0.05$; AUC glucose : AUC insulin 3.57, $p<0.05)$ followed by post hoc least significant difference test

cantly lower $(p<0.05)$, both on post-natal days 100 and 180. In keeping with high body fat percent, plasma triglyceride levels were significantly higher in offspring of vitamin-restricted mothers than in those of control mothers $(p<0.01)$ (Fig. 5) from post-natal day 70 onwards.

The rehabilitation of vitamin-restricted dams from parturition, but not from weaning corrected the changes almost completely by post-natal day 180 (Figs. 4, 5). Plasma total cholesterol and HDL cholesterol in the offspring were not significantly different among the groups at any of the time points tested (data not given).

Oxidative stress and antioxidant defense. Chronic maternal vitamin restriction resulted in a significant increase in MDA $(p<0.01)$ and a decrease in GSH levels $(p<0.05)$, but not in protein carbonyls (Table 4$)$ in the liver. Hepatic SOD and GPx activities were sig- 
Table 4. Indices of oxidative stress, antioxidants and antioxidant enzymes in liver homogenate of offspring of different groups on post-natal day 180

\begin{tabular}{lcccc}
\hline Parameter & VC & VR & VSP & VSW \\
\hline Malondialdehyde (nmol/mg protein) & $0.106 \pm 0.003^{\mathrm{a}}$ & $0.159 \pm 0.02^{\mathrm{b}}$ & $0.13 \pm 0.01^{\mathrm{ab}}$ & $0.102 \pm 0.007^{\mathrm{a}}$ \\
Protein carbonyls (nmol/mg protein) & $3.95 \pm 0.43$ & $4.80 \pm 0.43$ & $5.31 \pm 1.10$ & $5.00 \pm 0.40$ \\
Reduced glutathione ( $\mu$ mol/mg protein) & $2.85 \pm 0.24^{\mathrm{a}}$ & $1.83 \pm 0.26^{\mathrm{b}}$ & $2.94 \pm 0.24^{\mathrm{a}}$ & $3.03 \pm 0.41^{\mathrm{a}}$ \\
Antioxidant enzymes (U/mg protein) & & & \\
Catalase & $4.66 \pm 0.51$ & $4.69 \pm 0.51$ & $4.37 \pm 0.53$ \\
Superoxide dismutase & $14.88 \pm 0.41^{\mathrm{a}}$ & $17.68 \pm 0.27^{\mathrm{b}}$ & $14.32 \pm 0.34^{\mathrm{a}}$ & $17.60 \pm 0.57^{\mathrm{b}}$ \\
Glutathione peroxidase & $0.137 \pm 0.018^{\mathrm{a}}$ & $0.210 \pm 0.019^{\mathrm{b}}$ & $0.185 \pm 0.011^{\mathrm{b}}$ & $0.120 \pm 0.013^{\mathrm{a}}$ \\
\hline
\end{tabular}

Values represent means \pm SEM of 6 rats per group. VC, control diet throughout; VR, vitamin restriction throughout; VSP, vitamin supplementation to restricted mothers from parturition and to pups of such mothers from weaning; VSW, vitamin-restricted offspring weaned on to control diet. Means with different su-
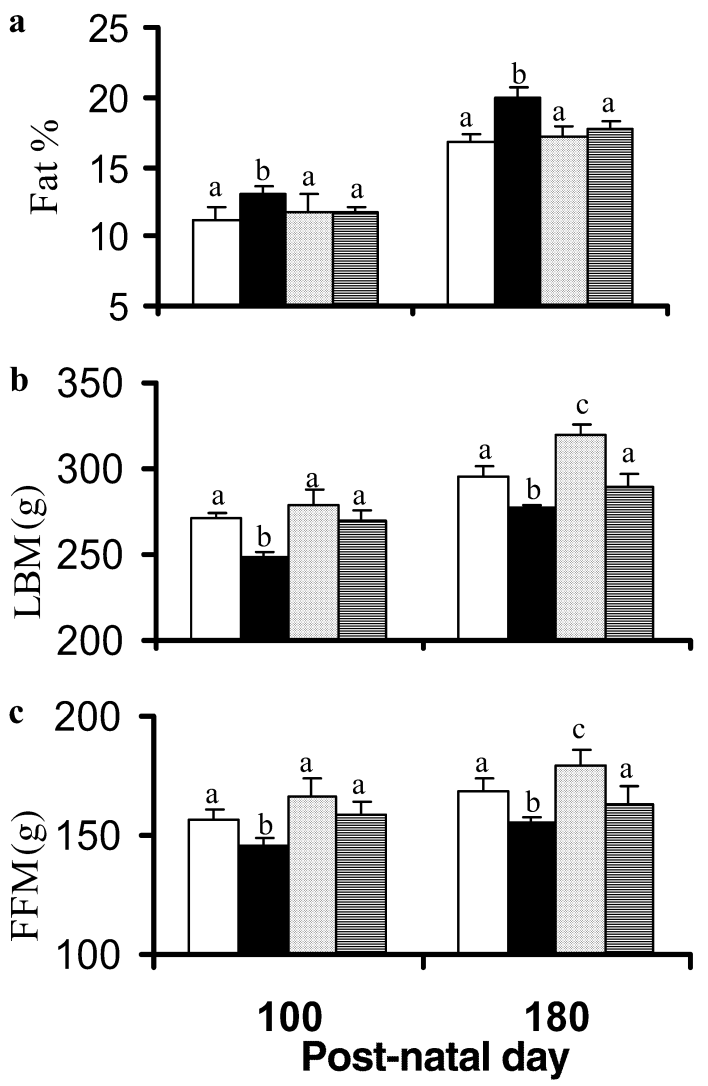

Fig. 4. Body fat percent (a), lean body mass (LBM) (b) and fat-free mass (FFM) (c) of the offspring on post-natal days 100 and 180, as determined by TOBEC. Open bars: control diet throughout; closed bars: vitamin restriction throughout; lightgrey bars: vitamin supplementation to restricted mothers from parturition and to pups of such mothers from weaning; darkgrey bars: vitamin-restricted offspring weaned on to control diet. Each bar represents a mean of observations in 6 rats. The line above each bar is the SEM. Means with different superscript letters are significantly different by one-way ANOVA $(F$ values at 100 and 180 days: body fat percent $3.32, p<0.05$ and 5.0, $p<0.05$, respectively; LBM 5.9, $p<0.01$ and 8.1, $p<0.001$, respectively; FFM 5.3, $p<0.01$ and $8.35, p<0.001$, respectively) followed by post hoc least significant difference test perscript letters are significantly different by one-way ANOVA ( $F$ values: malondialdehyde $6.04, p<0.01$; reduced glutathione $3.21, p<0.05$; superoxide dismutase $18.57, p<0.05$; glutathione peroxidase $7.21, p<0.05$ ) followed by post hoc least significant difference test

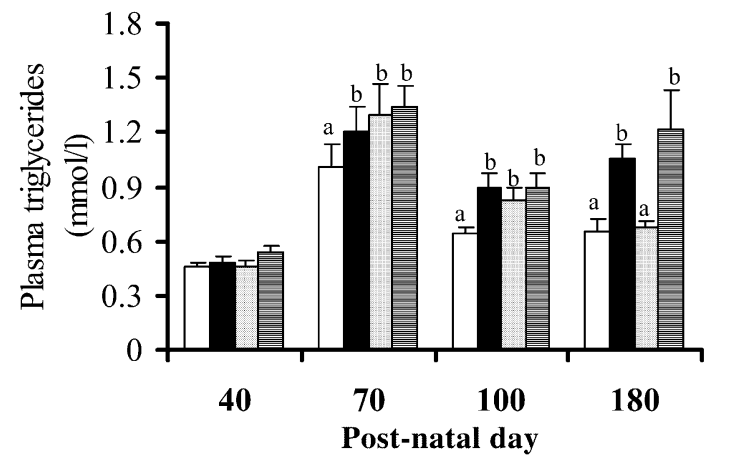

Fig. 5. Plasma triglycerides in the offspring of different groups on different post-natal days. Open bars: control diet throughout; closed bars: vitamin restriction throughout; lightgrey bars: vitamin supplementation to restricted mothers from parturition and to pups of such mothers from weaning; darkgrey bars: vitamin-restricted offspring weaned on to control diet. Each bar represents a mean of observations in 6 rats. The line above each bar is the SEM. Means with different superscript letters are significantly different by one-way ANOVA ( $F$ values: at 70 days $3.6, p<0.05$; at 100 days $3.88, p<0.05$; and at 180 days $5.84, p<0.05$ ) followed by post hoc least significant difference test

nificantly higher in vitamin-restricted offspring than in control offspring $(p<0.001)$ (Table 4). Rehabilitation either from parturition or weaning prevented the changes in MDA and GSH levels. The increased SOD was corrected by rehabilitation from parturition, but not by rehabilitation from weaning alone, whereas both appeared to correct the increase in GPx.

\section{Discussion}

Maternal undernutrition could predispose the offspring to diseases in adult life [3]. Although a number of animal models have been established to understand the mechanistic basis of this relationship [23, 24], most of them have dealt with the effect of maternal 
deficiency of macronutrients $[25,26]$. Little is known as to whether maternal dietary micronutrients, especially vitamins, play any role in the fetal origin of adult diseases. The present study was designed to assess the hypothesis that a $50 \%$ restriction of maternal dietary vitamins predisposes the offspring to insulin resistance syndrome in later life. We assessed the effect of maternal vitamin restriction during the mothers' growth, pregnancy and lactation on body weight, glucose tolerance, insulin resistance, body fat content and circulating lipid levels. We did this in the offspring on days 40, 70, 100 and 180 after birth.

Chronic maternal dietary vitamin restriction did not affect the birthweights of the offspring. Interestingly, only when maternal vitamin restriction was continued post-natally did it decrease the body weight of offspring at weaning and thereafter. By itself it did not decrease the offspring's body weight. The fact that these changes could be prevented by post-natal vitamin supplementation from parturition or weaning appears to stress the importance of neonatal and postnatal vitamin nutrition in the development of rat offspring. These findings are in partial agreement with earlier literature $[9,25]$.

Chronic, maternal vitamin restriction or its postnatal continuation in the offspring had no discernable effect on their fasting plasma glucose and insulin levels or oral glucose tolerance (AUC of glucose and insulin) till 6 months of age. Interestingly, on postnatal day 180, fasting plasma insulin levels as well as the AUC of insulin during OGTT were lower in all the groups compared with earlier time points, indicating developmental changes in the insulin status in rats and probably suggesting that a change in the glucose sensitivity of the rats occurs at this age. Despite these changes, no animal in any group had impaired oral glucose tolerance till 6 months of age, nor were the HOMA-IR values different among the groups. These observations are in agreement with Langley and coworkers $[27,28]$, who showed that maternal protein or iron restriction in rats affected glucose tolerance in the offspring only at 15 months but not at 3 months of age.

Notwithstanding the findings mentioned above, it was interesting that on post-natal day 180 , the AUC glucose : AUC insulin ratio during the OGTT was significantly higher in vitamin-restricted than in control offspring. These results indicate that maternal vitamin restriction and its post-natal continuation probably decrease the capacity of offspring to secrete insulin in response to an oral glucose challenge, and are in agreement with earlier literature showing that maternal protein deficiency had a similar effect on glucosestimulated insulin secretion in the offspring [27, 29, 30]. The fact that rehabilitation of vitamin-restricted mothers from parturition, but not rehabilitation of vitamin-restricted offspring from weaning, could mitigate this defect suggests the importance of neonatal and early post-natal vitamin nutrition in modulating/programming glucose-stimulated insulin secretion and hence glucose metabolism in the offspring.

One study [31] reported altered lipid metabolism in the pups of rat dams subjected to protein malnutrition or iron deficiency during pregnancy. Abundant literature indicates that altered body adiposity and/or lipid metabolism are the earliest changes seen, much before tissue insulin resistance becomes manifest [32, 33]. Indeed, it has been hypothesised that insulin resistance originates in impaired adipogenesis and/or lipid metabolism [33, 34, 35]. In line with these reports, offspring of vitamin-restricted mothers had higher body fat percent and plasma triglycerides along with lower lean body mass and fat-free mass than those of control-fed rats, probably suggesting that the former were developing insulin resistance. Interestingly, all these changes (with the exception of raised plasma triglyceride levels) were mitigated by rehabilitating vitamin-restricted mothers from parturition or vitamin-restricted pups from weaning, suggesting that maternal vitamin restriction and its post-natal continuation are relevant to the modulation/programming of the offspring's lipid metabolism.

The fact that only vitamin supplementation to vitamin-restricted mothers from parturition and to offspring of such mothers from weaning was able to correct the increased plasma triglyceride levels is comparable to their effects on the AUC glucose: AUC insulin ratio and highlights the importance of neonatal vitamin nutrition in modulating not only glucose but also lipid metabolism in the offspring. It remains to be seen whether the observed increase in plasma triglycerides is due to modulation of their clearance from the circulation and/or to lipolysis in tissues. In addition, the effects of neonatal vs post-weaning vitamin rehabilitation of vitamin-restricted pups on the modulation of these processes also needs to be determined.

There are reports that lipid abnormalities and insulin resistance are associated with an abnormal antioxidant defense and increased oxidative stress [36, 37]. It has also been suggested that oxidative stress plays a role in the development of insulin resistance [38, 39]. In line with these reports, we observed an increase in oxidative stress and decreased antioxidant status in vitamin-restricted rats compared with control rats. Given that some vitamins (e.g. Vitamin E) are very potent biological antioxidants, the increased oxidative stress in vitamin-restricted rats is not unexpected. As such, the increase in MDA and decrease in levels of reduced GSH in liver were also to be expected. Despite a significant increase in the activities of SOD and GPx in liver, vitamin-restricted rats had increased oxidative stress. This probably corroborates the importance of non-enzymatic antioxidants (especially vitamins) as the primary line of defense against oxidative stress [40] and suggests that maternal vitamin 
restriction modulates/programs antioxidant enzymes in the offspring to cope with the increased oxidative stress.

As a corollary to this, rehabilitation of vitaminrestricted mothers from parturition mitigated the changes in all the parameters mentioned above, stressing the importance of maternal vitamin restriction and its postnatal continuation in modulating oxidative stress in the offspring. However, in VSW pups, all parameters except hepatic SOD levels were mitigated. This is in line with our findings on the AUC glucose : AUC insulin ratios and plasma triglycerides levels, and underlines the importance of neonatal vitamin nutrition in reversing the changes induced by maternal vitamin restriction in the enzymatic anti-oxidant defense mechanisms of the offspring. Given that activities of antioxidant enzymes do not necessarily show a relation with oxidative stress and/or antioxidant status [40], the changes in the activities of antioxidant enzymes as expressed by the levels of hepatic MDA and GSH observed here may be acceptable.

Regardless of the mechanism(s) of modulation, it appears that the increased oxidative stress observed in maternal and/or post-natal vitamin restriction could be involved in the changes in adiposity and/or lipid metabolism, the well-recognised forerunners of insulin resistance.

In VSP and VSW animals, subsequent vitamin supplementation mitigated most of the changes in adiposity and lipid metabolism, as well as changes in oxidative stress and antioxidant defense. This probably suggests a causal relationship among these parameters, in addition to the relevance of maternal and postnatal vitamin nutrition of the offspring. To the best of our knowledge, this is the first report showing that chronic maternal vitamin restriction during the phases of mothers' growth, pregnancy and lactation affected the adiposity and lipid metabolism of the offspring and indicating that these effects could be due to increased oxidative stress and/or decreased antioxidant defense in the offspring. Furthermore, the high body fat percent and low body weight observed in vitaminrestricted offspring are similar to those reported in the "thin fat babies" in India, an abnormal condition attributed to maternal malnutrition [41] and thought to increase the risk of metabolic syndrome in later life. It thus appears from our results that the recent increase in the incidence of insulin resistance and its associated diseases among Indians could at least partly be due to widely prevalent micronutrient malnutrition and the resulting low-birthweight babies.

There are a number of similarities between our results and the effects reported in the offspring of mothers fed low protein diets [27, 31, 42]. Interesting$1 y$, in all these studies there was early growth retardation along with altered body composition and altered lipid profile. Although vitamin-restricted offspring had neither impaired glucose tolerance nor insulin re- sistance till post-natal day 180, the finding that they had significantly high body fat content with altered lipid metabolism (increased plasma triglycerides) probably suggests a predisposition to insulin resistance in later life. The rehabilitation of vitaminrestricted dams from parturition was more beneficial in preventing the changes in glucose and lipid metabolism and oxidative stress, than rehabilitating their pups from weaning. This, perhaps, stresses the importance of vitamin nutrition, especially during lactation, in predisposing offspring to metabolic syndrome in later life. Although we do not have direct evidence of insulin resistance, our results overall seem to support the hypothesis that maternal vitamin restriction predisposes the offspring to insulin resistance syndrome and associated diseases in later life.

Acknowledgements. The authors thank Dr Kamala Krishnaswamy, former Director, National Institute of Nutrition (ICMR), Hyderabad and Dr B. Sivakumar, Officer in charge, National Institute of Nutrition, for their interest in these investigations. We also thank Dr Ahamad Ibrahim for his useful suggestions in the preparation of the manuscript. L. Venu is thankful to the Council for Scientific and Industrial Research, India for the Junior Research fellowship award.

\section{References}

1. Ariyuki F (1987) Growth retardation induced in rat fetuses by maternal fasting and massive doses of ergocalciferol. J Nutr 117:342-348

2. Godfrey KM, Redman CW, Barker DJ, Osmond C (1991) The effect of maternal anaemia and iron deficiency on the ratio of foetal weight to placental weight. Br J Obstet Gynaecol 98:886-891

3. Hales CN, Barker DJ, Clark PM et al. (1991) Foetal and infant growth and impaired glucose tolerance at age 64. BMJ 303:1019-1022

4. Ramachandran A, Snehalatha C, Kapur A et al. (2001) Diabetes Epidemiology Study Group in India (DESI). High prevalence of diabetes and impaired glucose tolerance in India: National Urban Diabetes Survey. Diabetologia 44:1094-1101

5. Barker DJ (1994) Programming the baby. In: Barker DJ (ed) Mothers, babies and disease in the later life. BMJ Publishing Group, London, pp 14-36

6. Hales CN, Ozanne SE (2003) For Debate: Foetal and early post-natal growth restriction lead to diabetes, the metabolic syndrome and renal failure. Diabetologia 44:1094-1101

7. Hales CN (1997) Metabolic consequences of intrauterine growth retardation. Acta Paediatr [Suppl] 423:184-187

8. Anderson RA (2000) Role of dietary factors: micronutrients. Nutr Rev 58:S10-S11

9. Ashworth CJ, Antipatis C (2001) Micronutrient programming of development throughout gestation. Reproduction 122:527-535

10. Black RE (2001) Micronutrients in pregnancy. Br J Nutr 85:S193-S197

11. UNICEF (1998) The state of the world's children, 1998. Oxford University Press, Oxford and New York

12. Reeves PG (1997) Components of the AIN-93 Diets as improvements in the AIN-76A Diet. J Nutr 127:838S-841S 
13. Albala-Hurtado S, Novella-Rodriguez S, Veciana-Nogues MT, Marine-Font A (1997) Determination of vitamins A and $\mathrm{E}$ in infant milk formulae by high-performance liquid chromatography. J Chromatogr 778:243-246

14. Matthews JN, Altman DG, Campbell MJ, Royston $\mathrm{P}$ (1990) Analysis of serial measurements in medical research. BMJ 300:230-235

15. Morbach CA, Brans YW (1992) Determination of body composition in growing rats by total body electrical conductivity. J Pediatr Gastroenterol Nutr 14:283-292

16. Balasubramanian KA, Manohar M, Mathan VI (1988) An unidentified inhibitor of lipid peroxidation in intestinal mucosa. Biochim Biophys Acta 962:51-58

17. Uchida K, Kanematsu M, Sakai K et al. (1998) Proteinbound acrolein: potential markers for oxidative stress. Proc Natl Acad Sci USA 95:4882-4887

18. Hissin PJ, Hilf R (1976) A fluorometric method for determination of oxidized and reduced glutathione in tissues. Anal Biochem 74:214-226

19. Marklund S, Marklund G (1974) Involvement of the superoxide anion radical in the autoxidation of pyrogallol and a convenient assay for superoxide dismutase. Eur J Biochem 47:469-474

20. Aebi H (1984) Catalase in vitro. Methods Enzymol 105:121-126

21. Paglia DE, Valentine WN (1967) Studies on the quantitative and qualitative characterization of erythrocyte glutathione peroxidase. J Lab Clin Med 70:158-169

22. Schacterle GR, Pollack RL (1973) A simplified method for the quantitative assay of small amounts of protein in biologic material. Anal Biochem 51:654-655

23. Simmons RA, Templeton LJ, Gertz SJ (2001) Intrauterine growth retardation leads to the development of type 2 diabetes in the rat. Diabetes 50:2279-2286

24. Boloker J, Gertz SJ, Simmons RA (2002) Gestational diabetes leads to the development of diabetes in adulthood in the rat. Diabetes 51:1499-1506

25. Woodall SM, Johnston BM, Breier BH, Gluckman PD (1996) Chronic maternal undernutrition in the rat leads to delayed post-natal growth and elevated blood pressure of offspring. Pediatr Res 40:438-443

26. Langley SC, Jackson AA (1994) Increased systolic blood pressure in adult rats induced by foetal exposure to maternal low protein diets. Clin Sci (Lond) 86:217-222

27. Langley SC, Browne RF, Jackson AA (1994) Altered glucose tolerance in rats exposed to maternal low protein diets in utero. Comp Biochem Physiol Physiol 109:223229

28. Dahri S, Snoeck A, Reusens-Billen B, Remacle C, Hoet JJ (1991) Islet function in offspring of mothers on low-protein diet during gestation. Diabetes 40 [Suppl 2]:115-120
29. Swenne I, Crace CJ, Milner RD (1987) Persistent impairment of insulin secretory response to glucose in adult rats after limited period of protein-calorie malnutrition early in life. Diabetes 36:454-458

30. Merezak S, Reusens B, Renard A et al. (2004) Effect of maternal low-protein diet and taurine on the vulnerability of adult Wistar rat islets to cytokines. Diabetologia 47:669-675

31. Lucas A, Baker BA, Desai M, Hales CN (1996) Nutrition in pregnant or lactating rats programs lipid metabolism in the offspring. Br J Nutr 76:605-612

32. Smith U (2002) Impaired ('diabetic') insulin signaling and action occur in fat cells long before glucose intolerance-is insulin resistance initiated in the adipose tissue? Int J Obes Relat Metab Disord 26:897-904

33. Jones AP, Friedman MI (1982) Obesity and adipocyte abnormalities in offspring of rats undernourished during pregnancy. Science 215:1518-1519

34. Yajnik CS (2002) The lifecycle effects of nutrition and body size on adult adiposity, diabetes and cardiovascular disease. Obes Rev 3:217-224

35. Smith U, Axelsen M, Carvalho E, Eliasson B, Jansson PA, Wesslau C (1999) Insulin signaling and action in fat cells: associations with insulin resistance and type 2 diabetes. Ann NY Acad Sci 892:119-126

36. Urakawa H, Katsuki A, Sumida Y et al. (2003) Oxidative stress is associated with adiposity and insulin resistance in men. J Clin Endocrinol Metab 88:4673-4676

37. Katsuki A, Sumida Y, Urakawa H et al. (2004) Increased oxidative stress is associated with serum levels of triglyceride, insulin resistance and hyperinsulinemia in Japanese metabolically obese, normal weight men. Diabetes Care 27:631-632

38. Evans JL, Goldfine ID, Maddux BA, Grodsky GM (2002) Oxidative stress and stress-activated signaling pathways: a unifying hypothesis of type 2 diabetes. Endocr Rev 23:599622

39. Facchini FS, Hua NW, Reaven GM, Stoohs RA (2000) Hyperinsulinemia: the missing link among oxidative stress and age-related diseases? Free Radic Biol Med 29:1302-1 306

40. Vertuani S, Angusti A, Manfredini S (2004) The antioxidants and pro-oxidants network: an overview. Curr Pharm Des 10:1677-1694

41. Yajnik CS, Fall CH, Coyaji KJ et al. (2003) Neonatal anthropometry: the thin-fat Indian baby. The Pune Maternal Nutrition Study. Int J Obes Relat Metab Disord 27:173-180

42. Shepherd PR, Crowther NJ, Desai M, Hales CN, Ozanne SE (1997) Altered adipocyte properties in the offspring of protein malnourished rats. Br J Nutr 78:121-129 